few days after onset of measles infection are reported from the University of Catania, Italy. The cases occurred during measles epidemics in Italy (1988 and 1992) and the UK (1988). Spontaneous burning pain, increased by stimulation, or loss of pain sensation, over one side of the body was associated with mild ipsilateral paralysis. MRI showed bilateral swelling of thalamic areas and deep cerebral vein thrombosis. Symptoms remitted and MRI abnormalities resolved over a period of weeks or months. (Ruggieri M, Polizzi A, Pavone L, Musumeci S. Thalamic syndrome in children with measles infection and selective, reversible thalamic involvement. Pediatrics Jan 1998;101:112-119). (Respond: Martino Ruggieri MD, Division of Pediatric Neurology, Pediatric Clinic, University of Catania, 95125 Catania, Italy).

COMMENT. Acute measles infection may be complicated by deep cerebral venous thrombosis and symptoms of the thalamic syndrome. The symptoms can resolve without serious sequelae.

\title{
HIV-ASSOCIATED COGNITIVE DISORDERS AND CSF VIRAL RNA
}

The relationship of CSF levels of HIV type 1 RNA to neuropsychological

(NP) test performance in 97 prospectively enrolled, HIV-infected subjects (mean age, 31 yrs) was studied at the University of California, San Diego. In patients with AIDS, dementia and minor cognitive-motor disorders were associated with higher CSF RNA levels, whereas those without AIDS showed no relation between CSF RNA and NP impairment. In AIDS, CD4+ lymphocyte cell depletion $(<200)$ correlates with CNS HIV infection and dementia. (Ellis RJ, Hsia K, Spector SA et al, and the HIV Neurobehavioral Research Center Group. Cerebrospinal fluid human immunodeficiency virus type 1 RNA levels are elevated in neurocognitively impaired individuals with acquired immunodeficiency syndrome. Ann Neurol Nov 1997;42:679-688). (Respond: Dr Ellis, University of California, HIV Neurobehavioral Research Center, 2760 Fifth Ave, San Diego, CA 92103).

COMMENT. CSF viral studies link AIDS with cognitive impairments. HIV-1 RNA should be monitored in the CSF as well as plasma during antiretroviral therapy.

\section{LYME DISEASE AND FACIAL PALSY REVISITED}

The frequency of abnormal CSF findings in 40 children, ages 3-19 years, with new onset facial palsy and Lyme disease was determined at the State University of New York at Stony Brook. CSF white cell count, protein, or both were elevated in $68 \%$ of cases. The majority ( $89 \%$ ) had markers of B burgdorferi CNS invasion or immune involvement. Lyme disease-associated facial nerve palsy was related to CNS involvement and occult meningitis. Of 22 with CSF pleocytosis, none had meningeal signs and only one third had headache. (Belman AL, Reynolds L, Preston $\mathrm{T}$ et al. Cerebrospinal fluid findings in children with Lyme diseaseassociated facial nerve palsy. Arch Pediatr Adolesc Med Dec 1997;151:1224-1228). (Reprints: Anita L Belman MD, Department of Neurology, HCS T-12-020, State University of New York at Stony Brook, Stony Brook, NY 11794).

COMMENT. The diagnostic value of CSF examination in children with peripheral facial palsy and suspected Lyme borreliosis was reported from Zurich, Switzerland, in Neurology Sept 1997;49:817-824 (see Ped Neur Briefs Oct 1997). Analysis of CSF for specific antibodies to $B$ burgdorferi in children with acute facial palsy may facilitate eary diagnosis and prompt antibiotic treatment. 